\title{
ÖKOLOGISCHE SCHLUSSFOLGERUNGEN
}

\section{J. H. VAN VOORTHUYSEN}

Die Untersuchung des Ems-Estuarium mit dem Dollart und dem anschließenden Wattgebiet wurde u.a. vorgenommen, um durch das Sammeln von ökologischer Kenntnisse, die paläo-ökologischen Verhältnisse derartiger Regionen aus früheren Epochen der Erdgeschichte besser kennen zu lernen. Deshalb haben diese Schlußfolgerungen über die ökologischen Verhältnisse einen etwas anderen Akzent, als wenn sie von einem Biologen stammten.

Die Okologie der Diatomaceae, Mollusca, Ostracoda, Amphipoda, Copepoda, Foraminifera und noch einiger anderer wirbelloser Tiere wurde einer näheren Untersuchung unterzogen. Absichtlich war die Aufmerksamkeit auf die Mikrofauna und -flora gerichtet, weil wir besonders unsere mikropaläontologische Kenntnis vertiefen wollten.

Weiter wurde bewußt von dem Gedanken ausgegangen, nur rezente Ablagerungen mit ihrem biologischen Inhalt zu betrachten. Das gilt zum größten Teil auch für die sedimentpetrologischen Untersuchungen, wobei hauptsächlich der Sedimentationsmechanismus, wie dieser sich heutzutage abspielt, studiert worden ist.

Die Mittel, die uns für diese Arbeit zur Verfügung standen, erlaubten leider nicht, periodische ökologische Untersuchungen zu machen. Wir konnten nur während 3 Jahre im Sommer einige Wochen Feldarbeit verrichten. Die Kenntnisse der Ökologie der verschiedenen Pflanzen- und Tiergruppen hätten vertieft werden können durch periodische Untersuchungen während einiger Jahre. Hierdurch hätte sich die Anzahl von Arten, die heute zur Biocoenosis gerechnet worden ist, vielleicht noch etwas auf Kosten der Anzahl von Arten, die heute als zur Thanatocoenosis gehörig betrachtet wird, vergrößert; das allgemeine Bild der Mikrofauna hätte aber keine Änderung erfahren. Es zeigt sich nämlich die merkwürdige Tatsache, daß bei den Foraminiferen von
72 Arten mit Sicherheit nur 11 lebend angetroffen wurden, davon nur 5 Arten mit sehr viel Exemplaren pro Art. Trotzdem vermuten wir, daß sich zum Beispiel einige Arten der Gruppen Lagenidae, Miliolidae und Discorbininae, von denen keine lebend vorgefunden wurde, im untersuchten Gebiet behaupten können, wenn auch in wenigen Exemplaren pro Art. Die Möglichkeit, daß diese Gruppen sich aber im Frühjahr oder im Herbst vermehren, während nur ganz wenig Individuen sich im Sommer behaupten können, ist nicht ausgeschlossen. Sicher ist aber, daß die Exemplare (tot und lebend) von nur 5 Foraminiferenarten etwa $95 \%$ der Wattenfauna ausmachen.

Für die Ostracoden liegt die Sache einfacher. Es wurden insgesammt 8 lebende Arten im untersuchten Gebiet beobachtet, davon 6 in einer großen Anzahl von Exemplaren und 2 in einer geringen Anzahl; 28 Arten stammen aus der offenen Nordsee und werden nur als leere Schälchen im Wattgebiet angetroffen. Jedoch kann das Vorherrschen lebender juveniler Exemplare des Genus Leptocythere im Zusammenhang stehen mit ihren periodischen Entwicklungszyklus, so daß in den Sommermonaten noch keine erwachsenen Individuenen beobachtet werden können.

Über die Foraminifera und Ostracoda der offenen Nordsee sind noch relativ wenig exakte ökologische Angaben bekannt. Im allgemeinen können wir jedoch schon feststellen, daß das Wattgebiet mit dem sich darin befindenden Estuarium im Vergleich zu der offenen Nordsee arm ist an typischen Vertretern der litoral-innerneritischen Zone. Das konsequente Durchführen der Trennung zwischen Biocoenosen und Thanatocoenosen hat die obenerwähnte Tatsache deutlich dargelegt. Es stellte sich ja heraus, daß von vielen Foraminiferen und Ostracoden leere Schalen im Wattsediment angetroffen wurden, ohne daß nur ein 
einziges lebendes Exemplar vorgefunden wurde.

In der letzten Zeit ist man sich in der Mikrobiostratigraphie viel mehr bewußt geworden daß Verfrachtung von Fossilien und Verschleppung von rezenten schaletragenden Pflanzen und Tieren in eine andere Umgebung eine normale Erscheinung ist $\left.{ }^{\circ}\right)$. Bei ökologischen, paläo-ökologischen und stratigraphischen Arbeiten darf bei der Bestimmung eines Biotops diese Möglichkeit nicht vernachlässigt werden.

Für die Mollusca gilt diese allgemeine Regel aber nicht so scharf, denn im extremen Fall des untersuchten Wattgebietes mit dem sich darin befindenden Ems-Estuarium, kommen nur in der Nähe von Rottumeroog Mollusken aus einer anderen ökologischen Zone vor, nämlich dem litoral-innenneritischen Teil der offenen Nordsee.

Aus dem obenstehendem folgt, daß in einer fossilen litoral-lagunären Umgebung mit der Möglichkeit gerechnet werden muß, daß die vorgefundene Foraminiferen- und Ostracodenfauna z.T. aus einer anderen ökologischen Zone eingespiult worden ist, daß aber die Makrofauna im allgemeinen in situ angetroffen wird.

Untenstehende Tabelle gibt noch kein vollständiges Bild, denn von einigen Arten weiß man es bis jetzt noch nicht genau, ob sie überhaupt im Watt leben können. Sie zeigt jedoch eine deutliche Tendenz hinsichtlich der Verbreitung der Ostracoden, Foraminiferen und Diatomeen, nämlich die große Einseitigkeit in der Biocoenosis in Bezug auf die A tenzahl und die vielen aus einer anderen Umgebung verschleppten Arten, die im Wattmilieu nicht leben können.

-) Jones, Daniel, J. - Displacement of microfossils. Journ. Sed. Petr., Vol. 28, No. 4, S. 453-467, 1958.
Wir können im Ems-Estuarium zweierlei Thanatocoenosen unterscheiden:

le eine rezente synchrone Thanatocoenosis, aus einem anderen ökologischen Milieu herstammend;

2e eine fossile Thanatocoenosis (Paläothanatocoenosis).

Für die richtige ökologische Bestimmung kann die Nichtwiedererkennung einer verschleppten synchronen Thanatocoenosis zu falschen Schlußfolgerungen führen. Der SchluB, den wir aus dem obengesagten ziehen können, ist also, daß bei ökologischen Untersuchungen, vor allem der Mikroflora und fauna, es nicht genügt, nur die leeren Schalen zu erwähnen. Erstens miussen die lebenden und toten Individuen getrennt uerden. Zweitens ist eine derartige Untersuchung nicht vollständig, wenn nicht alle ineinander übergehenden ökologischen Zonen vom Süßwasser bis zur marinen Zone auf gleiche Weise untersucht worden sind.

Für die fossilen Thanatocoenosen von denen in den verschiedenen Beiträgen in dieser Abhandlung auch mehrere Beispiele genannt worden sind, liegt die Sache anders, aber bestimmt nicht einfacher. Besonders für die aufgearbeitete Mikrofauna und -flora der verschiedenen Gebiete, die zeitlich nicht so weit auseinander liegen, können große Schwierigkeiten entstehen, weil man hier den „älteren“ Charakter so schwer wiedererkennen kann. Dies ist besonders der Fall in der litoralen Zone an den Beckenrändern, wo kurze flache Transgressionen oft auftreten. Ein deutliches Beispiel ist die litoral-innerneritische Zone (von niedrigem Wasser bis $50 \mathrm{~m}$ Tiefe) des Nordseebeckens in jungtertiärer und altpleistozäner Zeit. In der außenneritischen (50-200 m Tiefe) und bathyalen

\begin{tabular}{|c|c|c|}
\hline & $\begin{array}{c}\text { Lebend angetroffene Arten im } \\
\text { Wattgebiet }\end{array}$ & $\begin{array}{c}\text { Verschleppte leere Schalen rezenter } \\
\text { Arten ats der offenen Nordsee im } \\
\text { Wattgebiet }\end{array}$ \\
\hline Ostracoden & $\begin{array}{c}\text { 6 } \\
\text { (in großer Anzahl von Individuen) } \\
2 \\
\text { (in geringer Anzahl von Individuen) }\end{array}$ & 28 \\
\hline Foraminiferen & $\begin{array}{c}5 \\
\text { (in großer Anzahl von Individuen) } \\
\pm 30 \\
\text { (in geringer Anzahl von Individuen) }\end{array}$ & \pm 37 \\
\hline $\begin{array}{l}\text { benthonisch: } \\
\text { Diatomeen } \\
\text { planktonisch: }\end{array}$ & $\begin{array}{c}54 \\
\text { (in großer Anzahl von Individuen) } \\
72 \\
\text { (in geringer Anzahl von Individuen) }\end{array}$ & 96 \\
\hline
\end{tabular}


Zone (über $200 \mathrm{~m}$ Tiefe) des marinen Raumes, besonders in Regionen, wo die Sedimentation während längerer Zeit gleichen Schritt mit der Senkung gehalten hat, sind "fossile" Beimischungen viel weniger zu erwarten.

Für die aufgearbeiteten Paläothanatocoenosen in einer Ablagerung, die, geologisch gesprochen, sehr viel jünger ist, ist die Sache weniger kompliziert. Ein gutes Beispiel bieten die in großer Anzahl im rezenten Wattsediment vorkommenden winzigen OberkreideForaminiferen. Sehr wahrscheinlich sind sie von der Kanalküste aus an den niederländischen und deutschen Nordseeküsten entlang transportiert worden. Derartige, geologisch viel ältere Fossilien, die von weit her verschleppt sein müssen, können uns, wie es hicr der Fall war, bei der Feststellung des Sedimentationsmechanismus helfen. Sie sind dann als Sedimentkörner zu betrachten und verhalten sich in ähnlicher Weise wie die Mineralfraktionen des Sedimentpetrologen.

Eine unerwartete Hilfe bei der lithologischen Bestimmung des Sedimentes war die Verbreitung einiger Amphipoda-Arten (Flohkrebse); es stellte sich heraus, $\mathrm{da} B$ diese stark abhängig ist von der granularen $\mathrm{Zu}$ sammensetzung. Auf Grund des Vorkommens einiger Arten dieser Crustaceen, die sich im Bodensediment eingraben, können wir ohne weiteres eine Aussage über die lithologische Beschaffenheit des Sediments machen. Arten, die zur Familie der Haustoriidae gehören, leben hauptsächlich in einem Sandboden mit einer granulometrischen Zusammensetzung, die etwa 70-80\% aus Körnern von mehr als $105 \mu$ besteht. Es ist sogar möglich, je nach den im Sediment befindlichen Arten, eine noch feinere Nuance der lithologischen Zusammensetzung anzugeben.

Weiter wurde untersucht ob ein Zusammenhang zwischen Bodenbeschaffenheit und Siedlung benthonischer Formen besteht. Wir müssen hier noch einigermaßen vag bleiben, weil die ökologischen Faktoren, die das Leben in diesem Estuarium bestimmen, sehr vielseitig und außerdem stark variabel sind. Im allgemeinen können wir doch sagen, daß die benthonischen Diatomeen sich wenig um die Bodenbeschaffenheit kümmern; Salzgehalt, Gehalt an organischem Stoff und Kalkgehalt des Bodens haben kaum Einfluß auf das Leben dieser Kieselalgen. Dann und wann tritt jedoch eine geringe Artverschiebung auf, wenn wir die Besiedlung von Schlick nach Sand verfolgen, aber das prozentuale Verhältnis von Süßwasser-, Brackwasser- und marinen Arten ändert sich nicht.

Für die Foraminiferen und Ostracoden gilt obengenannte Regel jedoch nicht. Die Arten dieser Gruppen hingegen sind mehr oder weniger stark abhängig vom
Gehalt an organischer Substanz des Sediments. Die Besiedlung von Ostracoden und Foraminiferen ist in den Uferschlicken mit ihren hohen Gehalt an organischem Stoff viel größer als auf den Sandplaten.

Aus obenstehendem darf die wichtige Schlußfolgerung gezogen werden, daß die in fossilen litorallagunären Ablagerungen befindlichen representativsten Ostracoden- und Foraminiferenfaunae in den an organischer Substanz reichen Tonen erwartet werden können. Diese Tatsache erklärt sich nicht nur dadurch, daß sie hier ihre günstigsten Lebensbedingungen vorfanden, sondern auch, da $\beta$ diese Tone in ruhigem Wasser abgelagert sind, wodurch die Möglichkeit einer eingespülten synchronen Thanatocoenosis sehr stark abgenommen hat.

In der Ems von der Dollartmündung stromaufwärts kommen bis auf eine Ausnahme, keine lebenden Brackwasser-Foraminiferen und Ostracoden vor. Diese Ausnahme betrifft Station Nr. 213 und 214, wo einige Brackwasser-Ostracoden bzw. Foraminiferen lebend angetroffen worden sind.

Es ist deutlich daß es einem zufälligen Umstande zuzuschreiben ist, daß einige Exemplare mit dem Bodengreifer noch lebend an die Oberfläche gekommen sind. Bewiesen ist damit, daß der Gezeitenstrom imstande ist, die lebende Mikrofauna viele Kilometer weit stromaufwärts zu verfrachten. Andererseits wird es aber nicht für wahrscheinlich gehalten, daß die ganze in der Ems bis Leer angetroffene tote Foraminiferengesellschaft vom Watt her stromaufwärts transportiert worden ist. Die Möglichkeit, da $B$ diese Thanatocoenosis teilweise aus den holozänen Tonen der Umgebung herstammt, ist m.E. sehr groß. Wir haben es dort sehr wahrscheinlich mit einer, zum größten Teil verschleppten, Paläothanatocoenosis zu tun.

Hinsichtlich des Vorkommens von Zwergformen in der Mikrofauna ist wenig Positives ans Licht getreten. Es ist sicher, daß wenigstens die Foraminiferen im allgemeinen eine etwas kleinere Gestalt besitzen, ohne daß wir hier von Nanismus sprechen dürfen. Nur zwei Foraminiferen-Arten des Genus Nonionella wurden als Zwergformen wiedererkannt.

An der Bestimmung der ökologischen Grenzen des Estuarium haben besonders die Diatomaceae, die Copepoda und die Amphipoda Anteil gehabt. Das Estuarium wird an der Landseite begrenzt durch eine Linie etwa $10 \mathrm{~km}$ stromaufwärts von Pogum an der E.ms, wo das Brackwasser in Süßwasser übergeht. An der Seeseite liegt die Grenze etwa am Nordpunkt der Hond Plate, wo die polyhalinen Arten verschwinden und ihr Platz wird durch pleio-mesohaline Formen eingenommen. 
Aus obenstehendem kann die Schlußfolgerung gezogen werden, daß die Verbreitung der lebenden Foraminiferen und Ostracoden an sich nicht genügt für die Bestimmung sehr exakter ökologischer Grenzen. Bei der Bestimmung paläo-ökologischer Grenzen, wobei beide letztgenannten Gruppen meistens eine wichtige Rolle spielen, muß darauf Rücksicht genommen werden.
Schließlich folgt unten eine kurze tabellarische Übersicht über die Verbreitung der wichtigsten untersuchten Tier- und Pflanzengruppen. Vier verschiedene ökologische Gebiete wurden unterschieden, während die Fauna und Flora in lebende, tote und fossile Exemplare aufgeteilt wurde.

Kurze Ubersicht der Verbreitung mehrerer Tier- und Pflanzengruppen (hauptsächlich Mikroorganismen) im untersuchten Gebiet

A. Süsswassergebiet der Ems

(von etwa $10 \mathrm{~km}$. östlich von Pogum stromaufwärts)

Lebend. (Biocoenosis)

\begin{tabular}{l|l} 
Foraminifera: & \\
& \\
Ostracoda: & keine \\
$\quad \begin{array}{l}\text { benthonisch: } \\
\begin{array}{l}\text { Diatomaceae } \\
\text { planktonisch: }\end{array}\end{array}$ & 63 Arten \\
$\begin{array}{l}\text { Thecamoebina: } \\
\text { Copepoda: } \\
\quad \text { Cyclopoida: }\end{array}$ & 36 Arten \\
$\begin{array}{l}\text { Cladocera: } \\
\text { Mollusca: }\end{array}$ & $\begin{array}{l}\text { Genus Cyclops } \\
\text { Fast alle Arten }\end{array}$
\end{tabular}

Tot. (Thanatocoenosis)

Vom Watt her stromaufwärts verfrachtet

Siehe Wagner's Beitrag in dieser Abhandlung

59 Arten

44 Arten

Difflugia sp. sehr wahrscheinlich noch mehrere Gattungen nicht nachweisbar nicht nachweisbar

\section{B. Der polyhaline und $\alpha$-mesohaline Teil des Ems-Estuariums} (etwa vom Nordpunkt Hond Plate bis Emden)

Foraminifera:
Ostracoda:
benthonisch:
Diatomaceae
planktonisch:
Ubrige wirbellose
Tiere
(Vgl. SchluBfol-
gerung Stock
und de Vos in
dieser Abhand-
lung)
Mollusca:

den genannten 8 Arten aus dem Dollartgebiet; fast völliges Fehlen lebender Exemplare in den Prielen

Ziemlich viele Arten te (ausgenommen Goesella waddensis). Elphidium gunteri bevorzugt aber das mesohaline Dollartgebiet.

8 als lebend erwähnte Arten

95 Arten

63 Arten

Gekennzeichnet durch das Fehlen bestimmter Arten. Optimale Verbreitung anderer Arten

zu den genanuten 8 Arten kommen noch hinzu: Littorina littorea, Retusa alba, Mysella bidentata, Petricola pholadiformis, Barnea candida
28 Arten (thanatocoenosis II von Wagner in dieser $\mathbf{A b}$ handlung)

79 Arten

43 Arten

nicht nachweisbar
Fossil. (Paläothanatocoenosis)

Ganz kleine Exemplare aus der Oberkreide. Verschleppte holozäne Formen aus der Umgebung

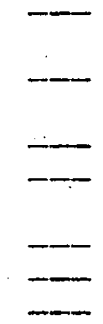

Ganz kleine Exemplare aus der Oberkreide (viel), normale Individuen aus dem Miozän, Altpleistozän und Eemien (wenig)

Keine deutlichen Anzeichen 
Foraminifera:

Ostracoda:

Mollusca:

Diatomaceae Amphipoda:

\section{Das $\alpha$ und $\beta$-mesohaline Dollartgebiet (litoral-lagunär)}

Lebend. (Biocoenosis)

Alle als lebend erwähnten Arten, aber Elphidium selseyensis bevorzugt das offene Wattgebiet. Goesella waddensis kommt nur im Dollartgebiet vor

Cyprideis torosa (nicht in sandiger Region)

Leptocythere castanea Leptocythere lacerta

benthonisch:

planktonisch:

\section{Arten}

99 Arten

Gammarus salinus (auch in der Ems) Corophium arenarium (fehlt im $\alpha$-mesohalin, tritt jedoch auf im $\beta$-mesohalin, Kerkeriet- und Reiderplate) Hydrobia ulvae (viel), H. ulvae forma I (viel), Macoma bal. thica (viel), Mya arenaria (viel), Scrobicularia plana (wenig), Cardium edule (wenig), Mytilus edulis (stellenweise), Retusa alba (sehr selten); fast völliges Fehlen lebender Exemplare in den Prielen
Tot. (Thanatocoenosis) Alle Arten, die als lebend und tot erwähnt sind. (Vgl. van Voorthuysen in dieser $\mathrm{Ab}$ handlung)

28 Arten, die nicht lebend im Wattgebiet angetroffen wurden (Vgl. Thanatocoenosis II von Wagner in dieser Abhandlung). 5 Arten, die nicht lebend im Dollart angetroffen wurden, aber wohl im offenen Wattgebiet

113 Arten

103 Arten

nicht nachweisbar

zu den hierneben genannten 8

Arten kommen ausnahmsweise noch hinzu:

Littorina littorea, Petricola pholadiformis, Barnea candida
Fossil. (Paläothanatocoenosis) Ganz kleine Oberkreide-foraminiferen (viel), normale lndividuen aus dem Miozän, Altpleistozän (wenig)

D. Das marine-polyhaline offene Wattgebiet (litoral-lagunär) (von den Nehrungsinseln Rottumeroog und Borkum bis etwa zum Nordpunkt Hond Plate)

Foraminifera: Ostracoda:

benthonisch:

Diatomaceae: planktonisch:

Copepoda:

Amphipoda:

Anisopoda:
Lebend. (Biocoenosis) Vgl..B (Ems-Estuarium) Vgl. B (Ems-Estuarium)

74 Arten

41 Arten

Leptinogaster histrio, Macrochiron fucicolum, Modiolicola insignis, Mytilicola intestinalis, Canuella furcigera, Pseudobradya beduina, Tisbe furcata

Corophium sextoni, Bathyporeia pelagica, Urothoe grimaldii

Tanaissus lillieborgi
Tot. (Thanatocoenosis) Vgl. B (Ems-Estuarium) Vgl. B (Ems-Estuarium)

24 Arten

36 Arten

nicht nachweisbar

nicht nachweisbar

nicht nachweisbar
Fossil. (Paläothanatocoenosis) Vgl. B (Ems-Estuarium) Ein Exemplar aus dem Jura (Uithuizer Watt) 
D. Das marine-polyhaline offene Wattgebiet (litoral-lagunär)

(von den Nehrungsinseln Rottumeroog und Borkum bis etwa zum Nordpunkt Hond Plate) (Fortsetzung)

Mollusca:

Echiuroidea:

Echinodermata:
Lebend. (Biocoenosis) zu den genannten 8 Arten aus dem Dollartgebiet kommen fast keine lebenden Arten hinzu; fast völliges Fehlen lebender Exemplare in den Prielen

Echiurus echiurus, (Sandplate bei Rottumeroog)

Asterias rubens Echinocardium cordatum
Tot. (Thanatocoenosis) zu den genannten 8 Arten kommen hinzu:

Mysella bidentata, Albra alba, Barnea candida, Angulus fabulus, Saxicavella jeffreysi, Donax vittatus, Montacuta ferruginosa, Hiatella arctica, und nach dem Norden zu: Littorina littorea, Ostrea edulis, Spisula subtruncata, Tellina tenuis

nicht nachweisbar

nicht nachweisbar
Fossil. (Paläothanatocoenosis)

11 Arten zur Eemienfauna gehörend

Station Nr. 317 in der Oude Westereems auf $15 \mathrm{~m}$. Tiefe) 


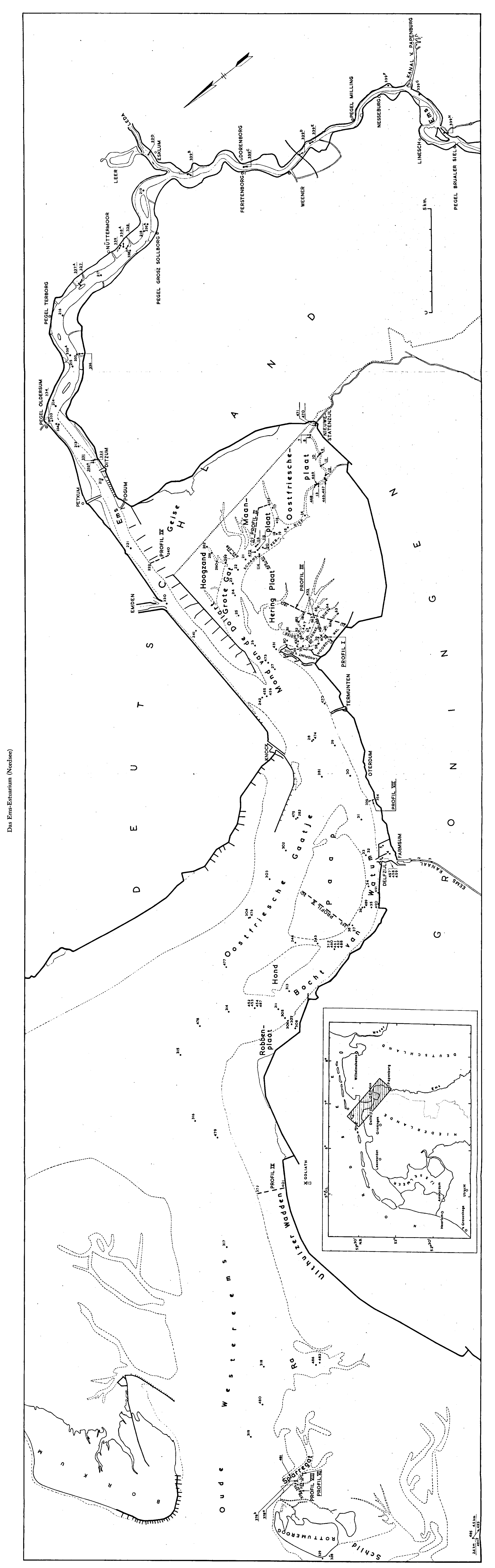

